\title{
Real-time Monitoring of the Horse-Rider Dyad Using Body Sensor Network Technology
}

\author{
D. Piette, T. Norton, V. Exadaktylos, and D. Berckmans
}

\begin{abstract}
When it comes to equestrian disciplines, the horserider dyad is amongst the most discussed topics. Recently the emergence of equitation science has led to an increased interest in objectively quantifying the interaction between the rider and horse. In this paper a methodology is presented to evaluate how the mental state of police horses interacts with that of their riders in order to assess the performance of police horses. This paper demonstrates how Body Sensor Network technology can be applied for real-time monitoring of the horse-rider dyad. The results of the study demonstrate that the mental state interaction between rider and horse is significantly different between bad police horses and good police horses.
\end{abstract}

\section{INTRODUCTION}

\section{A. The horse-rider dyad}

In equestrianism, the interaction between the rider and the horse is important. Rider and horse must work together in harmony in order to optimize not only their performance but also their health, safety and welfare. Lately, there is increasing interest in objectively quantifying and monitoring the horserider dyad by means of physiological measurements. This is partly the result of the recent emergence of equitation science, a discipline that aims at developing "scientific methods to study, measure and interpret interactions between horse and rider during equitation" [1].

This horse-rider interaction can be seen as twofold. First, there is the physical interaction which translates to for example riding style, riding aids, position and movement coordination between rider and horse [2,3]. Second, rider and horse interact in a nonphysical way. For instance, a rider can transfer his or her mental state to the horse, a phenomenon that is not only commonly known in the equine field but has also been confirmed in scientific literature [4, 5]. In Keeling's (2009) study for example, the rider was falsely told that the horse would be startled by an opening umbrella to make the rider nervous. Although the umbrella was never opened and the horse was not startled, the heart rate of the horse increased as a response to the nervous rider [5].

This paper demonstrates how the mental state of horses and their riders can be monitored in real-time using Body Sensor Network (BSN) technology, and how this information on mental state can be used to monitor and assess the horse-rider dyad.

\section{A. The Mounted Police}

The work presented in this paper is part of a larger study that was performed in collaboration with the mounted police in Brussels, Belgium. The scope of this study was to predict future performance of candidate police horses by monitoring their mental state as well as that of their riders. In particular, the negative mental state or stress of the horses and riders was monitored during their daily activities at the mounted police. The work presented in this paper is concerned with evaluating the horse-rider interaction during an obstacle test in an indoor arena, and relating this interaction to the performance of the horses.

\section{EXPERIMENTAL DESIGN}

\section{A. Subjects}

For the experiments, 9 police horses ( 3 mares and 6 geldings, aged 4-15 years) were divided into four categories by mounted police riders, based on their experience level and performance: good beginner, bad beginner, good experienced and bad experienced. Beginner horses are horses that have recently entered the training period at the mounted police. Experienced horses, in contrast, have been operational for several months up to several years. The label 'good' or 'bad' has been given to the horses by their riders based on previous experiences and based on the progress the horses have been making during their training period or operational career. The horses were ridden by 9 riders ( 4 female and 5 male, aged 24-50 years) of the mounted police. All measurements were performed twice with all but one horse, making sure that no horse was ridden twice by the same rider to take into account the effect of the rider on the horse.

\section{B. Body Sensor Network}

During the experiments the heart rate and physical activity of the horses were measured at a sampling frequency of $1 \mathrm{Hertz}$ using a Polar heart rate belt and a Zephyr module respectively. The heart rate and physical activity of the riders were measured using a Zephyr BioHarness at a sampling frequency of 1 Hertz. The heart rate was measured in beats per minute (bpm), the physical activity was measured in Vector Magnitude Units (VMU). Figure 1 demonstrates the BSN for the real-time monitoring of the heart rate and physical activity in rider and horse.

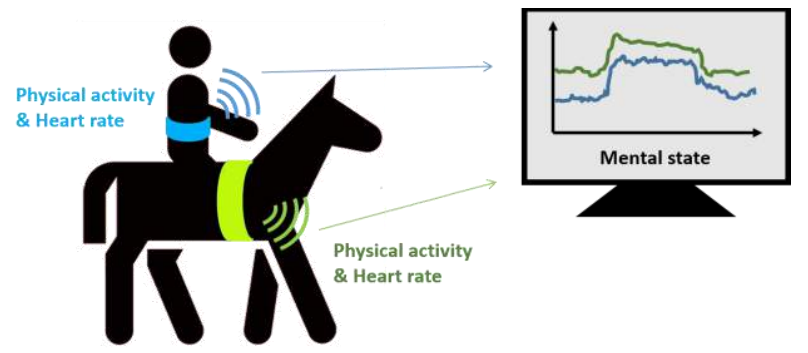

Figure 1: Illustration of the BSN used in the current study [6] 


\section{Experimental Protocol}

The experiments were performed in an indoor arena $(21 \mathrm{~m} \times 55 \mathrm{~m})$, which had a sand and fiber flooring material. During the experiments the horse-rider pairs performed an obstacle test following the protocol steps as described in TABLE I and illustrated in Figure 2.

TABLE I. EXPERIMENTAL PROTOCOL

\begin{tabular}{|c|l|}
\hline Step & Description \\
\hline $\mathbf{1}$ & The horse-rider pair stands still for 20 seconds \\
\hline $\mathbf{2}$ & $\begin{array}{l}\text { The horse-rider pair walks around in the arena at a steady pace } \\
\text { during 3 minutes }\end{array}$ \\
\hline $\mathbf{3}$ & The horse-rider pair walks over plastic bottles \\
\hline $\mathbf{4}$ & $\begin{array}{l}\text { The horse-rider pair walks through a half open curtain of plastic } \\
\text { straps }\end{array}$ \\
\hline $\mathbf{5}$ & $\begin{array}{l}\text { The horse-rider pair walks through two columns of stacked } \\
\text { barrels, the rider knocks over the upper barrel on his or hers } \\
\text { right hand side }\end{array}$ \\
\hline $\mathbf{6}$ & $\begin{array}{l}\text { The horse-rider pair stops at the speaker and remains standing } \\
\text { still while a sound recording (crescendo of applause, whistles, } \\
\text { gun sounds and explosions) lasting 20 seconds is being played }\end{array}$ \\
\hline $\mathbf{7}$ & $\begin{array}{l}\text { The horse-rider pair walks around in the arena at a steady pace } \\
\text { during 3 minutes }\end{array}$ \\
\hline $\mathbf{8}$ & End of the experiment \\
\hline
\end{tabular}

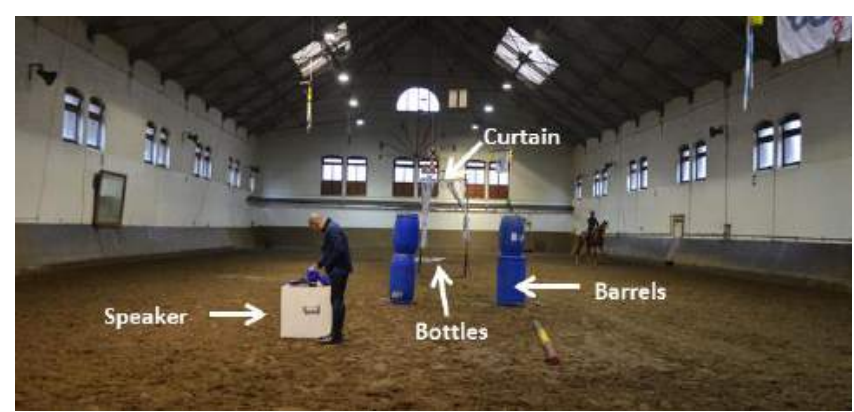

Figure 2: Picture of the indoor arena with obstacles

In total, the experiments were performed by 17 different horse-rider pairs. All experiments were recorded on video. The experiments were approved by the Animal Ethics Committee of KU Leuven for the measurements on horses and by the Social and Societal Ethics Committee of KU Leuven for the measurements on riders.

\section{Methodology}

The heart rate of a living organism can roughly be divided in three parts. A first part is related to the organism's metabolism, a second part is correlated with the physical activity of the organism and a third part links to the organism's mental state. This applies to horses as well as to humans. As previously mentioned this paper focusses on evaluating the negative mental state or stress in horses and their riders. An impression of how heart rate can change as a function of metabolism, physical activity and mental state is shown in Figure 3.

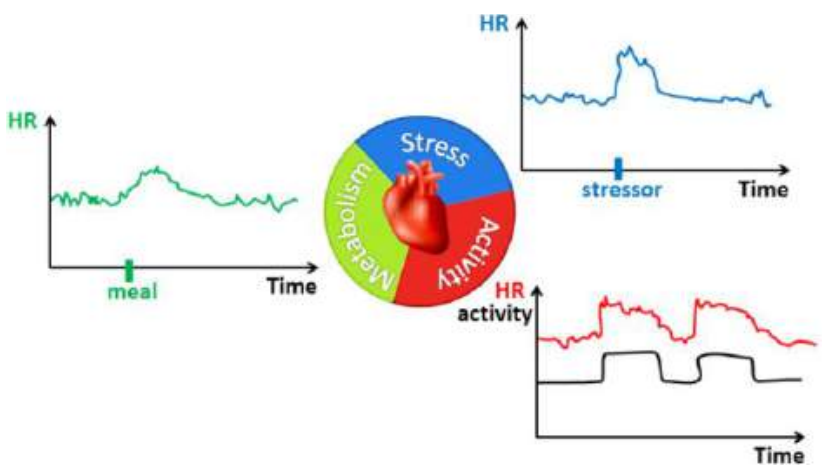

Figure 3: An impression of the three major determinants of heart rate [7]

In order to estimate the mental component of the heart rate, the components 'physical activity' and 'metabolism' have to be quantified. The physical activity is estimated by measuring the activity of the horses and riders. Given the short duration of the experiment, the metabolism is assumed constant during the experiments and is consequently not further taken into account.

Using the measured physical activity, a simulated heart rate was calculated that only reflects changes in heart rate resulting from changes in physical activity. This was done by applying a linear autoregressive model with exogenous input (ARX) of the second order with a time delay of one second to the measured physical activity. Model training was performed during step 1 and 2 of the protocol, when it can be assumed that horse and rider are in a neutral mental state. This resulted in a simulated or modelled heart rate describing the organism's heart rate component that is solely related to activity. This is in contrast to the measured heart rate which is also dependent on mental state. The positive difference between the measured heart rate and the modelled heart rate is a measure of the organism's mental state. After applying a 20 second window averaging filter to this difference and normalizing the result, a relative stress graph was obtained for every measurement. Figure 4 illustrates the different steps in the mental state detection. Validation of this methodology for mental state detection has been performed in previous work $[8,9,10]$.
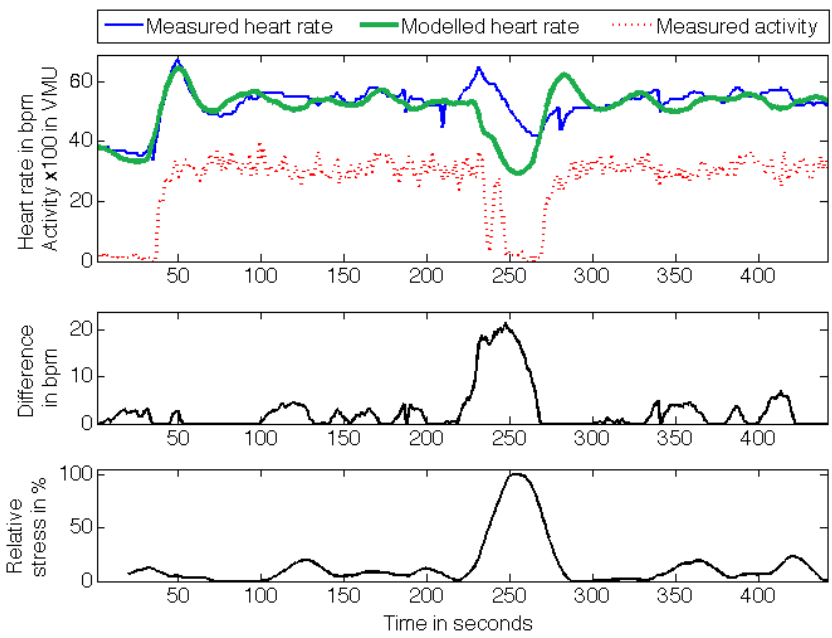

Figure 4: Illustration of the mental state detection. From top to bottom: modelled heart rate calculated based on physical activity, compared with measured heart rate - positive difference between measured and modelled heart rate - relative stress in \% after applying window averaging and normalization. 


\section{RESULTS AND DISCUSSION}

\section{A. Horse-rider interaction}

Of the 17 horse-rider pairs, eight pairs were removed from further analysis. Three pairs were rejected due to the measurement system failing to collect the heart rate data of the rider. Two pairs were omitted due to missing heart rate measurements in the rider. One pair was omitted due to inability to train the ARX model by the lack of a proper stepup in the rider's activity data. Two pairs were removed as a result of invalidated mental state detection in the rider. The horse-rider interaction of the remaining nine horse-rider pairs was assessed by comparing their relative stress graphs, as is illustrated in Figure 5. In this figure, it can be seen that the stress of both the rider and the horse peaks when the horserider pair is completing the obstacle course (between seconds 200 and 400). Another observation is that the stress of the rider starts increasing before the first obstacle is encountered (anticipation for the reaction of the horse). This observation was true for all horse-rider pairs. From the slope of the decrease in relative stress at the end of the obstacle course (step 6-7), it can also be seen that the rider seems to recover from the stressor more quickly than the horse.

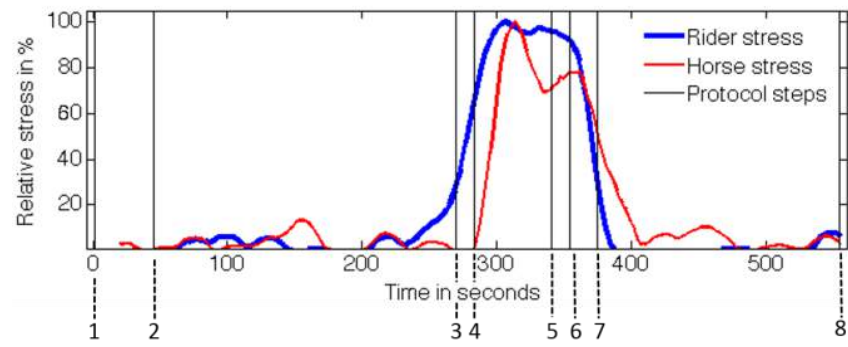

Figure 5: Comparison of the mental state of good beginner horse and its rider. The different steps in the protocol indicated in the figure are: 1) Standing still, 2) Walking, 3) Plastic bottles, 4) Curtain with plastic straps, 5) Rider knocks over a barrel, 6) Sound being played, 7) Walking, 8) End.

\section{B. Horse performance}

Further analysis of the interaction between the horse and its rider was performed by calculating the correlation between the relative stress graph of the rider and the relative stress graph of the horse. Figure 6 shows the relative stress graph of a good beginner horse and its rider. For this horse-rider pair the correlation between rider and horse stress is high, namely $86.09 \%$.

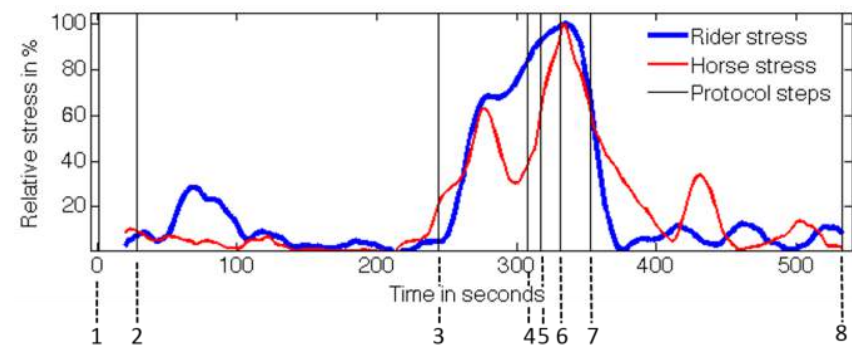

Figure 6: Comparison of the mental state of a good beginner horse and its rider. The different steps in the protocol indicated in the figure are: 1) Standing still, 2) Walking, 3) Plastic bottles, 4) Curtain with plastic straps, 5) Rider knocks over a barrel, 6) Sound being played, 7) Walking, 8) End.
Figure 7 shows the relative stress graph for a bad beginner horse and its rider. The correlation between the relative stress of this rider and horse $(44.39 \%)$ is lower than the one for the previously discussed horse-rider pair.

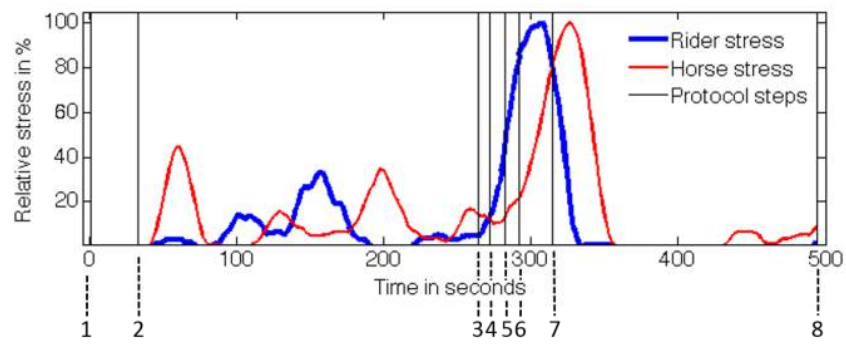

Figure 7: Comparison of the mental state of a bad beginner horse and its rider. The different steps in the protocol indicated in the figure are: 1) Standing still, 2) Walking, 3) Plastic bottles, 4) Curtain with plastic straps, 5) Rider knocks over a barrel, 6) Sound being played, 7) Walking, 8) End.

Based on these two examples, expectations are that the correlation of relative stress for rider and horse will be higher for good beginner horses compared to bad beginner horses. In order to investigate this, the correlation between the rider's and the horse's relative stress was computed for the nine horserider pairs. The results can be found in TABLE II. The first impression is that for beginner horses the correlation between rider and horse is indeed higher for good horses compared to bad horses, whereas the opposite is true for experienced horses. Thus, in spite of the very small sample sizes per horse category, an attempt was made to perform a multiple comparison test based on the correlation between the horse's and the rider's relative stress.

TABLE II. CORRELATION BETWEEN HORSE AND RIDER STRESS

\begin{tabular}{|c|c|c|}
\hline Horse-rider pair number & Horse category & Correlation \\
\hline $\mathbf{1}$ & Good beginner & 0.8609 \\
\hline $\mathbf{2}$ & Good beginner & 0.8880 \\
\hline $\mathbf{3}$ & Bad beginner & 0.4400 \\
\hline $\mathbf{4}$ & Bad beginner & 0.4439 \\
\hline $\mathbf{5}$ & Good experienced & 0.3937 \\
\hline $\mathbf{6}$ & Good experienced & 0.3788 \\
\hline $\mathbf{7}$ & Bad experienced & 0.8235 \\
\hline $\mathbf{8}$ & Bad experienced & 0.6280 \\
\hline $\mathbf{9}$ & Bad experienced & 0.6948 \\
\hline
\end{tabular}

In a first step the data in the four horse categories had to be tested for normality. Due to the small sample size, the JarqueBera test of normality with significance level $\alpha=0.05$ was conducted. The test resulted in p-values higher than the significance level $\alpha$, leading to the conclusion that the data in the four horse categories are coming from a normal distribution. Subsequently Barlett's test of equal variance was applied to the correlation data with significance level $\alpha=0.05$ which resulted in a p-value equal to 0.0535 . This indicates that the correlation values of the four horse categories have equal variance. A Tukey-Kramer test was then applied for multiple comparison with significance level $\alpha=0.05$ for the four different horse categories, giving the p-values listed in TABLE III. The pairwise comparisons for which the difference between both categories was found to be significant, are marked with an asterisk. 
TABLE III. P-VALUES OF THE MULITPLE COMPARISON TEST

\begin{tabular}{|c|c|c|}
\hline First category & Second category & p-value \\
\hline Good beginner & Bad beginner & $0.0040 *$ \\
\hline Good beginner & Good experienced & $0.0023 *$ \\
\hline Good beginner & Bad experienced & 0.1344 \\
\hline Bad beginner & Good experienced & 0.8176 \\
\hline Bad beginner & Bad experienced & $0.0194 *$ \\
\hline Good experienced & Bad experienced & $0.0089 *$ \\
\hline
\end{tabular}

What can be concluded from TABLE III is that based on the correlation between the relative stress of the rider and the relative stress of the horse, it is possible to distinguish between good and bad beginner horses as well as between good and bad experienced horses. Figure 8 visualizes the results of the multiple comparison test with confidence intervals. As can be observed from the figure, the $95 \%$ confidence intervals of the categories 'good beginner' and 'good experienced' show no overlap, as is the case for categories 'good experienced' and 'bad experienced'. Still, these results should be interpreted with care due the small sample size available for this analysis. Nevertheless they might demonstrate that the interaction between rider and horse is an important factor to be considered in the performance evaluation of police horses.

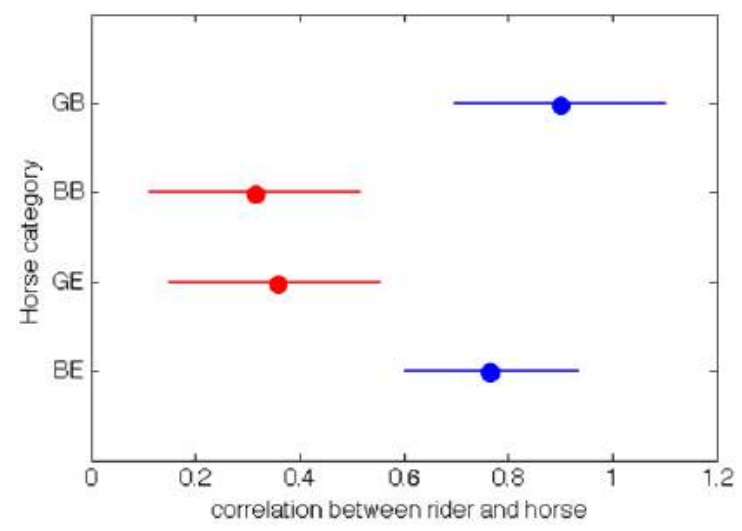

Figure 8: 95\% confidence intervals for the correlationg between the horse's and rider's relaive stress graphs for the different horse categories: good beginner (GB), bad beginner (BB), good experienced (GE) and bad experienced $(\mathrm{BE})$.

The fact that for beginner horses, the good horses show a higher correlation in relative stress with their rider whilst for experienced horses the opposite is true is rather counter intuitive. However a possible explanation could be that during the training phase horses that are focused on their rider and interact more with their rider will learn more and faster and are therefore good beginner horses. On the other hand, good experienced horses are supposed to know their job and be relatively comfortable in executing police tasks. Therefore they might need to rely less on their rider, which would explain the lower correlation between rider and horse for good experienced horses compared to bad experienced horses. Another explanation could be that the correlation between the relative stress of the rider and the horse gives valuable information on the performance of beginner horses, but simply does not for experienced horses.

\section{CONCLUSION}

This paper has demonstrated how BSN technology can be applied to monitor the mental state of horses and their riders in real time and that this methodology can be useful for the performance evaluation of police horses. It was found that good beginner police horses show a significantly higher correlation in mental state with their rider compared to bad beginner horses. Good experienced horses, however, show a significantly lower correlation in mental state with their rider compared to bad experienced horses. Despite the fact that these results should be interpreted with care due to the small sample size, they demonstrate the usability of horse-rider mental state interaction as a measure for performance in police horses.

Although this paper focused on police horses and police riders, it must be noted that the concept of mental state detection in horses and their riders can be extrapolated to other equestrian disciplines. Moreover, this methodology can also be applied in sports and disciplines that don't involve equids. For instance it could be used to monitor the interaction between humans or between humans and dogs.

\section{ACKNOWLEDGMENT}

The authors of this paper would like to thank the mounted police from Brussels, Belgium, for their collaboration and support throughout this project.

\section{REFERENCES}

[1] P. D. McGreevy, "The advent of equitation science," The Veterinary Journal, vol. 174, no. 3, pp. 492-500, 2007.

[2] M. Hausberger, H. Roche, S. Henry and E. K. Visser, "A review of the human-horse relationship," Applied Animal Behaviour Science, vol. 109, no. 1, pp. 1-24, 2008.

[3] I. A. Wolframm, J. Bosga and R. G. J. Meuleunbroek, "Coordination dynamics in horse-rider dyads," Human Movement Science, vol. 32, pp. 157-170, 2013.

[4] U. U. von Borstel, I. J. H. Duncan, A. K. Shoveller, S. T. Millman and L. J. Keeling, "Transfer of nervousness from competition rider to the horse," in Conference Proceedings $3 d$ International Equitation Science Conference, East Lansing, MI USA, Michigan State University, 2007, p. 16.

[5] L. J. Keeling, L. Jonare and L. Lanneborn, "Investigating horse-human interactions: the effect of a nervous human," Veterinary Journal, vol. 181, no. 1, pp. 70-71, 2009.

[6] ClipArt, "Figure of the horse-rider dyad," [Online]. Available: http://www.clipartbest.com/clipart-horseand-rider-silhouteet. [Accessed 2015].

[7] GH, "Figure of the human heart," [Online]. Available: http://galleryhip.com/human-heart.html. [Accessed 2014]. 
[8] V. Exadaktylos, F. Jansen, J. Van der Krogt, M. Guarino and D. Berckmans, "Real time monitoring of mental status of a horse to a negative stressor," in Precision Livestock Farming '13 ECPLF, Leuven, 2013.

[9] F. Jansen, J. Van der Krogt, K. Van Loon, V. Avezzu, M. Guarino, S. Quanten and D. Berckmans, "Online detection of an emotional response of a horse during physcial activity," Veterinary Journal, vol. 181, no. 1, pp. 38-42, 2009.

[10] D. Piette, "Real-time monitoring van mentale toestanden van paarden en ruiters," Master's Thesis, KU Leuven, 2015. 